\title{
Allons voir ailleurs si nous y sommes
}

Le défaut d'exotisme dans la prose postsoviétique

\section{Hélène Mélat}

\section{(2) OpenEdition}

\section{Journals}

Édition électronique

URL : http://journals.openedition.org/edl/436

DOI : $10.4000 /$ edl. 436

ISSN : 2296-5084

Éditeur

Université de Lausanne

Édition imprimée

Date de publication : 15 septembre 2009

Pagination : 241-252

ISBN : 978-2-940331-20-8

ISSN : 0014-2026

Référence électronique

Hélène Mélat, "Allons voir ailleurs si nous y sommes », Études de lettres [En ligne], 2-3 | 2009, mis en ligne le 15 septembre 2012, consulté le 19 décembre 2020. URL : http://journals.openedition.org/edl/ 436 ; DOI : https://doi.org/10.4000/edl.436 


\section{ALLONS VOIR AILLEURS SI NOUS Y SOMMES : LE DÉFAUT D'EXOTISME DANS LA PROSE POSTSOVIÉTIQUE}

Les auteurs de la Russie post-soviétique, Prigov, Bouïda, Chichkine, confrontés à nouveau, mais apparemment cette fois sans contraintes ni filtres politiques, à l'Occident et à l'Orient semblent découvrir un monde dont l'exotisme s'est déjà éventé; ils se lancent alors dans une quête dont l'objet est plus l'affirmation de soi que la rencontre avec l'Autre.

L'altérité est à la mode et l'exotisme en est l'une des facettes: à la distance indispensable à l'existence du concept d'altérité, s'ajoutent le désir, la fascination, le mystère. Pour Victor Segalen, l'exotisme est «la loi fondamentale de l'Intensité de la Sensation, de l'exaltation du Sentir; donc du vivre. C'est par la Différence, et dans le Divers, que s'exalte l'existence» ${ }^{1}$ et il est donc porteur, en plus d'une dimension sensuelle et émotionnelle, d'une valeur métaphysique. Qu'en est-il aujourd'hui pour l'écrivain voyageur russe au tournant $\mathrm{du} \mathrm{XXI}^{\mathrm{e}}$ siècle, à une époque où les frontières lui sont ouvertes? Existe-t-il dans la prose postsoviétique des lieux qui suscitent le désir et engendrent des projections fantasmatiques, des constructions mêlant présence du réel et pouvoir de l'imaginaire?

\section{La fatigue des "Europes»}

Beaucoup d'entre nous sont allés dans les différentes Europes. Plus moyen d'épater les copains avec ça. On a vu! on a assez vu! Qui peut-

I. V. Segalen, Essai sur l'exotisme, p. 92. 
on aujourd'hui intéresser en décrivant les mystères européens qui ne sont des mystères pour personne: ils sont connus ${ }^{2}$.

C'est par cette phrase que le narrateur bavard et fantasque de Mon Japon à moi seulement de Dmitri Prigov entame son récit sur l'Empire du soleil levant. De fait, l'Occident, après l'enthousiasme de la découverte du début des années 1990, n'occupe qu'une place modeste dans la fiction russe contemporaine et lorsqu'il est présent, il est le plus souvent banalisé. Distance réduite, culture assimilée ou ignorée ${ }^{3}$ : les effets de la globalisation se font sentir sur le terrain culturel aussi et l'on peut appliquer à la réalité d'aujourd'hui ce que Victor Segalen écrivait dès 1917 : «la Tension exotique du Monde décroît. L'Exotisme, source d'Energie, - mentale, esthétique ou physique [...], décroît " ${ }^{4}$.

Ce désenchantement de l'étranger est exprimé sur le mode comique par Evgueni Grichkovets: le héros de son monodrame En même temps (Odnovremenno, 1999) souffre d'une incapacité générale à savourer l'instant, ce qui s'exprime, entre autres symptômes, par le désir désespéré, mais impossible à assouvir, de ressentir la jouissance de l'exotisme devant l'objet rêvé et maintenant présent, comme, par exemple, la Madone Sixtine à Dresde. Les personnages des œuvres d'Oksana Robski, Ivan Tourguenev ou Anna Matveïeva ${ }^{5}$, lorsqu'ils séjournent à l'étranger, dirigent leur regard non vers les pays qu'ils traversent, mais sur eux-mêmes. La description du pays visité est quasi absente ${ }^{6}$, ce

2. «Mnogo naših nynče pobyvalo v raznyh Evropah. Rebjat ètim uže ne udivišs. Povidali! Kogo nynče poradueš’ opisaniem vseh vedomyh evropejskih nevedomostejoni izvestny", D. Prigov, Tol'ko moja Japonia. Nepridumannoe, p. 7.

3. Sans parler du rejet idéologique de tout Occident dans les écrits à tendance slavophile et nationaliste, qui par définition dénient au pays étranger tout attrait. Le pays étranger y est diabolisé et méprisé et cela ne laisse aucune place à l'exotisme.

4. V. Segalen, Essai sur l'exotisme, p. 93.

5. Cf. mon article «Le voyage en Europe dans la littérature russe postsoviétique», actes du colloque international «La Russie et l'Europe: autres et semblables», publication en ligne de l'Institut Est-Ouest, à paraître en 2009.

6. Natalia Ivanova remarque cette même rareté des paysages et une «absence principielle d'impressions" à propos d'un essai de Boris Ryji, Le Journal d'Amsterdam [Amsterdamskij dnevnik] et souligne dans son article l'enfermement de l'écrivain russe sur son propre moi. N. Ivanova, «Victime de la géographie: l'écrivain russe découvre et referme le monde», p. 301. 
dernier n'étant qu'une toile de fond, un simple décor réduit à quelques clichés, n'ayant pas de valeur en soi et n'éveillant aucune curiosité.

Dans Le Cheveu de Vénus (Venerin volos, 2004) de Mikhaïl Chichkine, émigré en Suisse 7 , le champ spatial dépasse largement l'Europe, puisqu'il englobe le Moyen-Orient (la Perse) et le GrandNord sibérien. L'Europe, bien qu'opposée en tous points à la Russie, selon les modalités classiques (calme/violence, intensité/ platitude, pauvreté/ richesse), est pourtant nivelée avec cette dernière dans la mesure où, au cœur de l'univers fictionnel de Chichkine, l'équivalence est le rapport qui, selon les lois du postmodernisme, gère toute chose et en particulier les textes, littéraires ou non: l'auteur postule que toutes les histoires sont les mêmes, en conséquence tous les lieux se rejoignent et perdent leur charge exotique. Rome, par exemple, sous la surface et les clichés touristiques n'est qu'une ville faussaire hantée par la mort et l'inauthentique, ses statues ne sont que des copies inertes rappelant les corps alignés des victimes de la guerre de Tchétchénie, dont l'auteur énumère les noms comme dans un rapport de police ou une litanie d'âmes perdues. Ce principe d'équivalence entraîne l'interpénétration et la convergence finale des lignes narratives: les combats de la Perse antique, tout droit sortis de L'Anabase de Xénophon, opposent Grecs, Perses et Tchétchènes tandis que Daphnis et Chloé deviennent les héros des cosmogonies orotche et toungouze. Réintégrer la culture russe dans le fleuve d'une culture plus vaste se fait au prix de l'indifférenciation et de l'annulation de l'exotisme.

Dans son essai La Suisse russe (Rousskaïa Chveïtsaria, 1998), Chichkine, quittant les voies du romanesque, semble redonner à la Suisse, synecdoque de l'Europe, son caractère exotique en relatant les séjours d'hommes célèbres russes depuis le XVIII e siècle et leurs impressions. Le recul du temps et le parti pris initial du documentaire permettent, dans un premier temps, de rétablir la distance annulée dans son œuvre de fiction. D'emblée, l'auteur insiste sur l'étrangeté de la Suisse pour le Russe, mais les signes s'inversent sans tarder:

"Que dire de la Suisse? Des paysages de carte postale et rien d'autre, au point que je commence à en avoir la nausée et que, si je me trouvais

7. Chez les écrivains émigrés, le pays d'accueil suscite rarement le désir, mais représente plutôt un pis-aller qui ne peut par essence être l'objet d'un discours amoureux. 
en ce moment au milieu de notre nature sans beauté ni relief, avec ses isbas de rondins et son ciel grisâtre, je serais capable de m'en émerveiller comme d'un paysage original.» Ça, c'est $\mathrm{Gogol}^{8}$.

Chichkine désexotise l'Europe par une vision qui rend la Suisse quotidienne, voire triviale, et la russifie: "Gogol grave son nom sur la pierre de la prison de Chillon", "Rozanov essaie le fauteuil de Calvin" et "Chagall fait paître à Zurich les vaches de Vitebsk " ${ }^{9} .$. Chichkine s'inscrit ici dans le mouvement général de démythification du passé et de ses grands hommes très à la mode dans la Russie de la fin du XX $\mathrm{XX}^{\mathrm{e}}$ siècle. Mais de ce fait, la Suisse aussi se trouve démythifiée.

L'Amérique, dans Le grand Pays (Velikaïa strana, 2001) de Leonid Kostioukov, est l'objet d'une satire souriante, mais malgré l'exotisme d'une langue truffée d'américanismes, ce roman est fondamentalement placé sous le signe d'une dualité réduisant la distance entre les deux pays: le héros, Maggie/ David, est un transsexuel également transfrontalier, puisque c'est un Juif russe. Le "grand pays» du titre peut désigner la Russie tout aussi bien que l'Amérique: là encore, c'est le principe d'équivalence plus que de différence qui régit l'économie du texte.

On peut néanmoins trouver quelques rares îlots d'exotisme, par exemple Yermo de Iouri Bouilda. Venise, où se déroule l'action, y est l'écrin baroque d'une histoire semi-policière et dans le reflet infini et trompeur de ses miroirs redoublés se love un mystère constitutif de l'exotisme. L'évocation de Venise relève ici de la technique de l'orientalisme qui maquille et déguise son objet. Ville des masques et du carnaval, des ors et du carmin, à l'instar d'un joyau précieux, la Venise de Bouïda oblitère toute mention de la Russie et garde intacte sa puissance d'objet du désir:

8. “Čto Tebe skazat' o Švejcarii? Vse vidy da vidy, tak čto mne uže ot nih nakonec stanovitsja tošno, i esli by mne popalos' teper' naše podloe i ploskoe russkoe mestopoloženie s brevenčatoju izboju i seren'kim nebom, to ja byl by v sostojanii im voshiščat'sja, kak novym vidom”. Eto Gogol'", http://magazines.russ. ru/inostran/1998/9/shishkin.html (tous les sites mentionnés dans cet article ont été consultés en septembre 2008). Edition française: M. Chichkine, La Suisse russe, p. 13.

9. "Gogol vycarapyvaet svoe imja na kamnjah šil'onskoj tjur'my», "Rozanov usaživaetsja v kreslo Kal'vina», "Šagall prigonjaet v Cjurih vitebskih korov», http:// magazines.russ.ru/inostran/1998/9/shishkin.html. M. Chichkine, La Suisse russe, p. 10 . 
Le cœur battant de joie, il s'était arrêté au centre d'une petite place devant la pesante façade du palazzo Sanseverino [...] La maison de ses rêves [...]. Dans son enfance, elle lui apparaissait par bribes: des colonnes, des frontons, des galeries, des algues touffues tapissant les murs à la surface de l'eau, des pigeons sur les anges qui décoraient le toit... Plus tard, en rêve, il avait réussi à pénétrer à l'intérieur, à gravir un large escalier flanqué de larges rampes blanches qui se recroquevillaient au bout en vases-coquillages, avec, au fond, de vivantes fleurs épicées, il avait réussi à pénétrer dans des salles avec de petits anges bien léchés à la Bellini, des tableaux de Palma et de Paris Bordone, des clairs-obscurs d'Ugo de Caroi, avec des portes aux vitrages craquelés ouvrant sur une galerie; c'était là que l'attendait, et le pressentiment du bonheur se faisait de plus en plus fort et de plus en plus joyeux : cette porte, là, ensuite, à gauche, encore une porte, et enfin - elle: vêtue d'une robe vaporeuse d'un blanc rosé, la tête tournée, en train de courir, tout essoufflée, riant aux éclats, avec des cheveux roux défaits et des yeux bleus étonnés $[\ldots]^{10}$.

L'exotisme ici est sensuel: Venise est la métaphore du corps féminin, et l'exaltation est d'autant plus forte que la progression vers le saint des saints est longue. Au bout de la route, il y a l'autre, objet exotique suprême, en l'occurrence la femme.

Io. «S radostno b'juščimsja serdcem on ostanovilsja v centre malen'koj campi, pered tjaželovesnym fasadom palacco Sanseverino [...] Dom iz ego snovidenij [...] V detstve on javlialsja emu po kusočkam - kolonny, frontony, galerei, mahristye vodorosli, oblepivšie steny u sreza vody, golubi nad angelami, ukrašavšimi kryšu... Pozdnee emu udavalos' - vo sne - proniknut' vnutr', podnjat'sja po širokoj lestnice s širokimi belymi perilami, na koncah skručivavšimisja v rakoviny-vazy s živymi prjanymi cvetami v glubine, vojti v zaly s zalizannymi angeločkami à la Bellini, kartinami Palmy i Parisa Bordone, k’aroskuro Ugo da Carpi, s krakeljažnymi steklami v dverjah, vyvodjaščimi na galereju, - a vperedi ždalo glavnoe, i on znal, dogadyvalsja, čto ego ždet, otčego predčuvstvie sčastia stanovilos' sil'nee i radostnee, - v tu dver', teper' nalevo, ešče odna dver', nakonec - ona: v belo-rozovom vozdušnom plat'e, v poloborota, na begu, zadyhajuščajasja, smejuščajasja, s razmetavšimisja ryževatymi volosami i udivlennymi golubymi glaziščami...], Ermo, in Skoree oblako, čem ptica [Plutôt un nuage qu'un oiseau], Edition française: I. Bouïda, Yermo, p. 9-10. 


\section{A l'Est rien de nouveau?}

Venise, comme le remarque Bouïda, est aux portes de l'Orient, apparemment plus chargée d'exotisme que l'Occident dans la prose postsoviétique, riche en références à la spiritualité orientale: le bouddhisme, par exemple, permet aux personnages de Viktor Pelevine de trouver leur "Mongolie intérieure» "11 dans 2008 (2006) de Sergueï Dorenko, le héros, Vladimir Poutine en personne, s'est entiché de taoïsme. Le voyage reste abstrait, mais lorsqu'il se réalise concrètement il peut être encore représenté comme un rituel d'initiation. Le héros du Tao du saxophoniste (Dao saksofonista, 2003) d'Andreï Batov, le saxophoniste du titre, personnage passif appartenant au type du héros de conte, en est un exemple. Le récit de son voyage vers la Chine, où des mafieux l'emploient à jouer dans un hôtel, relève du pur roman d'aventure, la péripétie la plus exotique étant le sauvetage, dense en action, de l'énorme camion qui le transporte et est enseveli sous la neige. Etranger signifie démesure: en témoigne la torture qu'infligent au héros ses employeurs - l'emprisonnement dans une caisse froide tapissée de clous qui lui meurtrissent la chair. Il sortira de ces épreuves réellement transformé. Mais le voyage exotique peut n'être qu'une fausse initiation, comme dans Travel Agneau (Travel agnec, 1999) d'Anastassia Gosteva, dont la narratrice découvre, cinq siècles après Colomb, non pas l'Amérique, mais l'Inde et ce, trente ans après les hippies des années 1970. Presque entièrement consacré à l'état d'esprit dépressif et aux délires haschischo-acides de son héroüne, le roman présente une Inde réduite à la portion congrue, filtrée et singulièrement amaigrie par la perception d'une jeune Moscovite plutôt passive. Dans les deux tiers du roman, les héros sont confinés dans une chambre d'hôtel de New Delhi, cette diminution de l'espace étant la métaphore de leur enfermement mental. Les voyages artificiels évoqués dans les dialogues à bâtons rompus qui entrecoupent régulièrement le récit de la narratrice sont totalement dénués d'exotisme et ne représentent aucunement l'espace mystique qu'on serait en droit d'attendre. Le voyage n'a de valeur initiatique que dans la mesure où l'héroïne comprend en fin d'ouvrage qu'elle doit trouver les réponses à ses interrogations chez elle...

II. Pour plus de détails, cf. mon article «De la Mongolie intérieure à l'Empire britannique: les nouveaux horizons de la littérature russe grand public», p. 239-256. 


\section{Un exotisme littéraire}

La matière du texte lui-même serait-elle le dernier refuge de l'exotisme? Boris Akounine, par exemple, offre au lecteur russe deux types de personnages nouveaux pour lui : le dandy Eraste Fandorine, modèle européen, marqué au coin d'un double exotisme, puisqu'à partir du roman Léviathan il apparaît en compagnie du Japonais Massa, qui devient son fidèle serviteur. Ils constituent un couple comique, par le contraste physique (l'un est mince, l'autre gros) et le décalage qu'apporte l'étrangeté du Japonais. Le fait de flanquer le personnage d'Eraste, type classique, d'un personnage totalement opaque, régénère les clichés. Les romans policiers de Kholm van Zaïtchik, dont l'action est située au milieu du XXI siècle en Ordouss, pays fictif, mélange eurasien de Russie et de Chine, sont placés d'emblée sous le signe de la mystification, puisqu'ils sont présentés comme étant l'œuvre d'un lettré hollandais ayant choisi d'écrire uniquement en chinois. A l'appui de cette déclaration, les vrais auteurs ${ }^{12}$ décorent leurs textes de nombreux caractères chinois, ajoutent des notes d'un traducteur fictif, une biographie fictive, un article critique en postface et de nombreuses références intertextuelles ${ }^{13}$.

C'est aussi un exotisme littéraire que Prigov met en ouvre dans sa trilogie placée sous le signe des lieux (Moscou, le Japon puis la Chine) avec laquelle, selon ses propres paroles, il a voulu «essayer les trois types européens d'écriture de la sincérité: les Mémoires, le récit de voyage et la confession " ${ }^{14}$. Le lecteur est donc en présence d'un projet littéraire où il n'est guère question de "sincérité »... Le narrateur de Mon Japon à moi seulement, double parodique de l'auteur, dérape sans arrêt dans son récit, dérivant dans des digressions variées, dont bon nombre réactualisent son passé de gamin des rues moscovites, pauvre et affamé - comme, d'ailleurs, dans Katia la Chinoise (Katia kitaïskä̈a, 2007),

I2. Un sinisant, Igor Alimov (1964), et l'auteur d'œuvres fantastiques Viatcheslav Rybakov (1954).

I3. La référence à Robert van Gulik, implicite dans l'ensemble de l'œuvre, devient explicite dans Le Juge Ti, où c'est un chat qui porte le nom de l'illustre limier fictif.

I4. «Vse tri romana - èto kak by ispytanie treh tipov evropejskogo iskrennego pis'ma - memuarov, zapisok putešestvennika i ispovedi», D. Prigov, A. Jahontova, "Othody dejatel'nosti central'nogo fantoma" [Les Déchets de l'activité du fantôme central], http://magazines.russ.ru/nlo/2004/65/prig16.html. 
où alternent son point de vue et celui d'une fillette. On retrouve ici le regard centripète observé plus haut: le pays exotique est le miroir du moi du narrateur et n'existe qu'en fonction de ces retours en arrière. Le narrateur insiste plusieurs fois sur le fait que tous les pays se ressemblent et sur la monotonie des voyages, annulant de facto la nécessité du récit de voyage qui devient ainsi une parodie dénonçant les clichés liés à la vision du Japon; et par ce biais Prigov déconstruit le mythe japonais de la littérature russe, comme le signale Alexandre Tchvantsev ${ }^{15}$. Pourfendre les clichés, c'est l'une des occupations favorites de l'artiste conceptualiste, on le sait, et cette révision de l'image du Japon passe par sa "désexotisation» et une "désorientalisation» esthétique: les réalités décrites sont les cimetières, les toilettes, la nourriture, autant d'éléments opposés à la vision idéalisée d'un tableau orientaliste. La cérémonie du thé, par exemple, sommet de raffinement de la culture japonaise, est présentée sous un angle de vue ironique et presque grotesque. Voici ce qu'en dit le narrateur après la description longue (sur une page et demie) et précise du rituel vu de l'extérieur:

Pendant tout le laps de temps long et parfois pénible que je viens de décrire, l'hôte et contemplateur de cette cérémonie extrêmement solennelle et respectable à un point rare doit rester assis sans bouger. Sans trahir par un seul muscle son impatience ou son inconfort. Et c'est bien comme cela qu'il est assis. Et tout cela, rappelons-le, pour une malheureuse petite tasse de thé $[\ldots]^{16}$.

Cette mise à distance du genre du récit de voyage fait écran à la réception du Japon, qui n'est plus que l'outil d'un jeu littéraire. Elle centre l'intérêt sur le narrateur, et la forme du discours adressé, relevant du $s k a z$, renforce le comique et oriente la lecture vers l'image et les petites histoires du gamin moscovite et de sa bande. Katia la Chinoise propose une vision nivelée de la réalité chinoise, présentée du point de vue d'une enfant, pour laquelle l'exotisme n'a pas encore de sens, tout étant encore

I5. Aleksandr Čvancev, «Iz Japonii v molčanie» [ Du Japon au silence»], http:// magazines.russ.ru/nlo/2007/87/.

I6. Ves' vyšeopisannyj dlitel'nyj i poroj mučitelnyj vremennoj promežutok gost' dolžen sidet' bez dviženija. Ni edinym muskulom ne vydavaja svoego neterpenija ili že neudobstva. I on sidit imenno takim obrazom. I vse eto, napomnim, iz-za odnoj-edinstvennoj čašečki čaja [...], p. 65. 
découverte. La "défamiliarisation" (l'ostranenie chklovskienne) produit finalement une «familiarisation» du monde chinois, Prigov ne gardant de l'exotisme pour le lecteur, qui suit les découvertes de l'héroïne, que celui des monstres qui viennent régulièrement visiter la petite fille, le premier étant le dragon d'un défilé de Nouvel An.

\section{L'Ultima Thule de l'exotisme}

Il semble paradoxal que la Russie s'ouvrant après soixante-dix ans de régime communiste soit si peu perméable à l'exotisme et que l'écrivain russe s'empresse de retourner au point de départ, chez lui. L'exotisme apparaît repoussé aux marges de la fiction, dans la prose documentaire, comme dans le roman-essai L'Ile. Eloge des voyages insensés de Vassili Golovanov. L'Ultima Thule de Golovanov, c'est le bout du monde russe, l'île Kolgouïev, dans la mer de Barents, où le narrateurauteur-journaliste, héritier des géologues des années 1950-1970, les "derniers romantiques de l'utopie soviétique", selon l'expression d'Igor Chevelev ${ }^{17}$, part en quête d'une "percée existentielle dans un autre espace/ temps ${ }^{18}$ et du sens de la vie. Il se définit comme un fugitif, et la matière du livre est constituée de l'évocation de cette fuite, entrecoupée de réflexions, puis de la transformation de cette fuite en expédition organisée: à partir de la fuite se construit une nouvelle vision de l'autre et de soi, sur la base d'une destruction - celle du rêve, car l'île, ravagée à l'ère postsoviétique par la misère et l'alcoolisme, ne correspond plus à la vision idéalisée transmise au narrateur par le livre des deux peintres à l'origine de son voyage. Le narrateur de Golovanov dépasse la déception, "transforme les pas en mots», le voyage en matériau littéraire - ce qu'il considère être son devoir envers l'Ile. Ce n'est pas un hasard si le narrateur emporte partout un appareil photo et que ce soient deux peintres qui aient alimenté son rêve: le héros du livre, c'est l'espace du Grand-Nord. L'exotisme est lié ici non seulement aux nombreux

I7. I. Ševelev, «Ostrova v okeane» [«Les îles de l'océan»], http://newshevelev.narod. ru/genkat/846.htm

I8. «èkzistencial'nyj proryv v inoe prostranstvo/vremja», V. Golovanov, Ostrov. Opravdanie bessmyslennyh putešestvij, p. 10. Edition française: Eloge des voyages insensés, traduit par H. Châtelain. 
éléments ethnographiques dont l'auteur émaille son texte, mais aussi au passage entre deux entités opposées de l'univers qui régissent la vie humaine - le passage du temps à l'espace. La pesanteur que le narrateur ressent le besoin de quitter, c'est la tyrannie du temps, mesure de la vie urbaine, qui fait oublier l'espace, mesure de la vie naturelle.

Dans toutes les œuvres évoquées, on est loin de l'idéalisation et de l'orientalisme et la superposition du pays d'origine et de l'étranger, indissociable de l'exotisme, est majoritairement centripète, dirigée vers l'origine: "Voyager dans un pays, quel qu'il soit, équivaut à voyager en Russie» ${ }^{19}$. Pourquoi un tel défaut d'exotisme? "Ne peuvent sentir la Différence que ceux qui possèdent une Individualité forte. [...] ceux-là goûteront pleinement l'admirable sensation, qui sentiront ce qu'ils sont et ce qu'ils ne sont pas» ${ }^{20}$, estimait Victor Segalen. Le voyageur russe, tel qu'il est représenté dans la prose postsoviétique, a une identité en pâte à modeler, brouillée, qui risque constamment de se désintégrer au contact d'une dimension étrangère, aussi se rend-il imperméable à cette autre dimension. Seuls quelques-uns dépassent véritablement les frontières: Bouïda parce qu'il est originaire d'une région excentrée, multiethnique et multiculturelle, la Prusse orientale, et que l'Autre (en particulier l'Allemagne) est un composant à part entière de sa culture; Golovanov parce qu'il transcende ses propres frontières et débouche, suivant ses propres termes, dans un "espace mystique» certes intimement lié à l'espace de l'île, mais qui est dans le même temps un espace de liberté universel.

Dans la majorité de la littérature russe du tournant du XXI siècle, l'Autre, si exotique qu'il soit, est essentiellement un instrument annexé à la contemplation de soi: c'est sa propre image, torturée et traumatisée, que la Russie cherche à cerner aujourd'hui dans le miroir de la fiction, fascinée par l'auto-exotisme narcissique du lancinant "Je est un autre».

Hélène MÉLAT

Paris

I9. «Poezdka v ljubuju stranu okazyvaetsja poezdkoj v Rossiju» : C'est ce qu'écrit Chichkine à propos de Tchaadaïev, dans La Suisse russe, p. 12 de la traduction en français.

20. V. Segalen, Essai sur l'exotisme, p. 43. 


\section{BIBLIOGRAPHIE}

Bujda, J., Ermo, in id., Skoree oblako, čem ptica [Plutôt un nuage qu'un oiseau], Moskva, Vagrius, 2000 (1996). Ed. fran. Yermo, tr. par S. BeneCh, Paris, Gallimard, 2002.

Čvancev, A., «Iz Japonii v molčanie» [«Du Japon au silence»], http://magazines.russ.ru/nlo/2007/87/.

Golovanov, V., Ostrov. Opravdanie bessmyslennyh putešestvij, Moskva, Vagrius, 2002. Ed. fran. Eloge des voyages insensés, tr. par H. Châtelain, Lagrasse, Verdier, 2007.

Ivanova, N., «Victime de la géographie: l'écrivain russe découvre et referme le monde", Cahiers slaves, 2008.

MÉlat, H., "De la Mongolie intérieure à l'Empire britannique: les nouveaux horizons de la littérature russe grand public», Slavica Occitania, 11 (2000).

—, "Le voyage en Europe dans la littérature russe postsoviétique", Actes du colloque international «La Russie et l'Europe: autres et semblables", publ. en ligne de l'Institut Est-Ouest, à paraître en 2009.

Prigov, D., Tol'ko moja Japonia. Nepridumannoe, Moskva, NLO, 2006. Prigov, D., Jahontova, A., "Othody dejatel'nosti central'nogo fantoma" ["Les déchets de l'activité du fantôme central»], http://magazines.russ.ru/nlo/2004/65/prig16.html

Segalen, V., Essai sur l'exotisme, (Biblio essais), Paris, Le Livre de poche, 1978.

Ševelev, I., «Ostrova v okeane» [«Les îles de l'océan »], http://newshevelev.narod.ru/genkat/846.htm

ŠIšKIn, M., "Urok švejcarskogo», Inostrannaja literatura, 9 (1998), http ://magazines.russ.ru/inostran/1998/9/shishkin.html. Ed. fran., La Suisse russe, tr. par M. Fellous, Paris, Fayard, 2007. 
